\title{
M etáforas evaluativas del alumnado universitario de Educación Física. Necesidad de un cambio radical Evaluative metaphors of the university students of Physical Education. Need for a radical change \\ María Amparo Calatayud Salom \\ Universidad deValència (España)
}

\begin{abstract}
Resumen. Lafinalidad de este artículo es analizar las metáforas que mejor han radiografiado la evaluación que los estudiantes universitarios de Educación Física han vivido a lo largo de su escolaridad. Para ello, se ha contado con una muestra de 275 estudiantes universitarios de Educación Física de varias universidades públicas de la ComunidadValenciana. Los resultados señalan, desde la mirada de los estudiantes, que la evaluación vivida dista mucho del paradigma educativo centrado en la eval uación formativay en la retroalimentación. Constatar estarealidad es necesario para darse cuentade queéstahade cambiar si queremos realizar prácticas evaluativas de excelencia que estimulen la generación del aprender a aprender y del uso de la eval uación como fuente y al servicio del aprendizaje. Todo ello nos lleva a señalar que los docentes universitarios tenemos el reto de formar a futuros profesores de Educación Física, más críticos y reflexivos y que, especialmente, sean promotores del cambio y la innovación evaluativa en las aulas cuando ejerzan como profesionales de la educación. Por tanto, este articulo ofrece la oportunidad de cambiar la cultura eval uativaimperante aún, hoy, en los centros educativos através de los argumentos y propuestas que se proponen.
\end{abstract}

Palabrasclave: Evaluación; aprendizaje; mejora; metáforas, innovación.

Summary. The purpose of this article is to analyze the metaphors that have best radiographed the evaluation that university students of Physical Education have experienced throughout their schooling. To this end, a sample of 275 Physical Education university students from various public universities in theValencian Community has been counted on. The results indicate, from the point of view of the students, that the lived evaluation is far from the educational paradigm focused on formative evaluation and feedback. Verifying this reality is necessary to realize that it has to change if we want to carry out excellent eval uation practices that stimulate the generation of learning to learn and the use of eval uation as a source and at the service of learning. All this leads us to point out that university teachers havethe challenge of training future Physical Education teachers, who are more critical and reflective and who, especially, are promoters of change and evaluative innovation in the classroom when they work as education professionals. Therefore, this article offers the opportunity to change the evaluative culture that still prevails today in educational centers through the arguments and proposal s that are proposed.

Keywords: Evaluation; learning; improvement; metaphors, innovation.

\section{A modo de introducción}

La evaluación suscita un gran interés entre los teóricos, investigadores, estudiantes, profesorado y familia. Como señalan Anijovic, y Cappelletti (2017), evaluar es una tarea ardua que condensa sentidos construidos desde el sistema educativo y que define la trayectoria escolar de los estudiantes. Además, se trata de una práctica que impacta en el estudiante, en la familia y en la propia institución escolar.

Durante nuestro trabajo de formación de futurosprofesores de distintos niveles educativos en la U niversidad hemos realizado varios estudios que tratan de pro-

Fecha recepción: 13-08-20. Fecha de aceptación: 22-02-21

Mariam Calatayud Salom

amparo.calatayud@uv.es fundizar en el tema de la evaluación de los aprendizajes de los estudiantes (Cal atayud, 2019; Cal atayud, 2019a). En este estudio, se ha pretendido indagar a cerca de las metáforas que durante la escolaridad ha desencadenado el tema de la evaluación. Cuando nos referimos al concepto de metáforahemos querido hacer al usión alalente que nos permite ver la realidad de las prácticas evaluativas en la etapa escolar. Desde esta realidad podremos penetrar en la complejidad de las representa ciones evaluativas.

Según el diccionario de la Real Academia de la Lengua, la metáfora es la figura retórica de pensamiento por medio de la cual una realidad o concepto se expresa por medio de una realidad o concepto diferente con el que lo representado guarda cierta relación de semejanza. Por tanto, se ha utilizado este recurso literario para desvelar las representaciones que el al umnado univer- 
sitario de Educación Física tiene en relación con la eva luación.

Indagar sobre la evaluación vivida nos ha permitido acercarnos un poco a la cultura evaluativa que aún impera en la actualidad y, especialmente, darnos cuenta de que a pesar de la emergencia y de los cambios continuos, la praxis evaluativa no ha cambiado. Se sigue eva luando de la misma manera y la mayoría de las metáforas acerca de la evaluación vivida contienen connotaciones negativas y muy tradicionales. M etáforas que suelen incidir en el modelo de evaluación tradicionalsumativo en Educación Física que suele centrarse en cómo obtener la calificación de los estudiantes al final de un trimestre o curso y en el que suele predominar la utilización de test de condición física y/ o habilidad motriz.Y en las que la nota final del estudiante procede de los resultados obtenidos en dichos test 0 al menos un porcentaje importante de la misma. (López-Pastor, 1999; López-Pastor et al.,2013; Rodríguez-Gómez. y Ibarra-Sáiz, 2015; M acPhail \& Murphy, 2017)

Es por ello que este articulo pretende ser una aportación más en este necesario trabajo de aproximación a la realidad de la evaluación desde el punto de vista de los estudiantes universitarios, no sólo para conocerlo y comprenderlo, sino para analizar eintentar cambiar con argumentos aquellos aspectos de la evaluación que no incidan en una concepción de la evaluación como una oportunidad para el aprendizaje y en la creencia del error como fuente también de aprendizaje. En Educa ción Física es necesario avanzar hacia una cultura de la evaluación centrada en sistemas de evaluación alterna tiva que se aproximan al modelo de evaluación formativa y compartida y/ o evaluación para el aprendizaje (Hernán et al.,2020: López-Pastor et al.,2016; Rodríguez-Gómez y Ibarra-Sáiz, 2015).

Revisando la literatura internacional especializada (Richard y Godbout, 2000; Casbon y Spackman, 2005; Hay, 2006; MacPhail y Hal bert, 2010, Lorente y Kirk, 2013; N i Chróinín y Cosgrave 2013; Tolgfors y Ö hman, 2015; Leirhaug y M acPhail, 2015; Mac Phail y M urphy, 2017; López-Pastor y Sicilia-Camacho, 2017), se puede apreciar claramente como la evaluación formativa en Educación Física ha ido evolucionando hacia enfoques que, como comentan López Pastor et al., (2020), están más centrados en el proceso de aprendizaje del alumnado, y en las que los términos como: «evaluación alternativa», »evaluación para el aprendizaje», «evalua ción orientada al aprendizaje» 0 «evaluación auténtica» entre otros términos, impregnan más la teoría que las prácticas. Vocablos que se sumergen en los parámetros de laTradición Educativa de la evaluación.

Laformación en evaluación es fundamental para que los profesores de Educación Física en formación adquieran una cultura evaluativa impregnada en supuestos formativos que puedan generar después con sus estudiantes. Una formación inicial que desarrolle buenas competencias en evaluación porque, sin lugar a dudas, como señalaPerrenoud (2004), laeval uación es unacompetencia docente básica y también una de las más costosas y tediosas de la función docente, que genera miedos y reticencias, hasta el punto de que se trata de uno de los aspectos en el que más cuesta cambiar e innovar y, en dónde, es necesario invertir más formación para que el profesorado comprenda el sentido genuino de la eva luación.

\section{Cultura evaluativa imperante en el escena- rio actual}

Hoy por hoy, en muchos de nuestros Institutos de Educación Secundaria de España, aún persiste la cultura de la evaluación dirigida casi exclusivamente a medir resultados finales de aprendizaje. La función social de la eval uación ha sido mucho más determinante que la función educativa, de análisis de los procesos, de diagnóstico, de comprensión, diálogo, retroalimentación y de participación activa del alumnado en el proceso evaluativo. Seguimos sometiendo a nuestros estudiantes a pruebas que sólo estimulan la memorización, la repetición, el pensamiento convergente, etc. y nos olvidamos que lo que, en estos momentos, es relevante, es el desarrollar habilidades para la resolución de problemas, la capacidad crítica, la necesidad de que los conocimientos previos sirvan de unión a los nuevos conocimientos a fin de que cada estudiante genere su propia significación personal de lo aprendido. Concepción de la evaluación vinculada directamente con la valoración de los resultados del aprendizaje de los estudiantes y mediatizada por una racionalidad propia de la perspectiva tecnoburocrática del currículum. Y en la que la eva Iuación se sitúa en un pensamiento restringido: sumativo y cuantificador. Es lo que autores como López Pastor et al. (2016) han denominado como lacultura del examen. Algunos supuestos de esta concepción evaluativa que hemos denominado como Examinadora y que surgen de investigaciones recientes (Calatayud, 2019, 2019a) inciden básicamente, en aspectos que tienen que ver con:

-Prevalece la observación de los resultados más que la valoración de los procesos. 
-Sólo se evalúan contenidos conceptuales.

-Evaluación comprobativa (si los objetivos previstos se han al canzado).

-Evaluación continua con carácter sumativo.

-La evaluación como instrumento coercitivo, punitivo, etc.

-Evaluación finalista.

- U so casi exclusivo del examen.

-El culpable del fracaso escolar es el alumno.

- 0 bjeto de evaluación es exclusivamenteel discente.

-No se negocian los criterios de evaluación con los alumnos.

-La recuperación es la repetición del examen.

-Evaluar es sinónimo de calificar, medir, etc.

-Se menosprecia el uso de la autoevaluación como elemento de reflexión, desarrollo y madurez.

- La referencia al control/ examen para tomar las decisiones de promoción.

-En las sesiones de eval uación se «cantan» las notas.

-No se asume la importancia del error como fuente de aprendizaje.

-Evaluación para detectar lo negativo.

-Evaluación puntual.

-Evaluación con carácter explícito.

-Se les otorga más peso a las evaluaciones de tipo formal.

-Se ignora el valor de la evaluación del proceso de enseñanza y aprendizaje como instrumento de mejora de la Institución Escolar.

Por tanto, se trata de una concepción evaluativa que incide en la relevancia de certificar 0 cal ificar los aprendizajes, basada en pruebas orales y escritas con pautas de observación rígidas que se aplican al final izar la unidad didáctica, en las que se sanciona el error. Además, no le preocupa el contexto en el que ocurre el aprendizaje, distribuye a los estudiantes en jerarquías de excelenciay el discente esagente pasivo del proceso de aprendizaje.

Esta forma de evaluar hoy por hoy no tiene ningún sentido. Desconocemos si el estudiante es capaz de relacionar lo aprendido con otros conocimientos, si es ca paz de trasladarlo a situaciones distintas a aquellas que generaron el aprendizaje original, si es competente, etc. Como ya se ha demostrado sobradamente, hoy en día, no tiene sentido la acumulación de información, la información está en internet, bien organizada, categorizada y de calidad. EI docente del siglo XXI ha de evolucionar para pasar de ser un mero transmisor de la información a facilitador de criterios en la búsqueda de la información y de su transformación en conoci- miento y aprendizaje (González-Sanmamed et al., 2018). Lo relevante es que los estudiantes adquieran información, la integran, la procesen, sean creativos, intuitivos, emprendedores, críticos y protagonistas de su proceso formativo.

\section{Evaluación como oportunidad para apren- der}

Los avances por abordar una concepción de la eva luación que apuesta por insertarlaen el proceso de aprendizaje, primar la función de diagnóstico, de regulación y adaptación de la planificación del proceso de enseñanza y aprendizaje a las necesidades y dificultades de los estudiantes, dista mucho de la realidad que acontece en nuestros centros educativos.

Hoy por hoy, la evaluación debería de ser entendida como señalan Anijovich y Cappelletti (2017) como una oportunidad para que los estudiantes pongan en juego sus saberes, visibilicen sus logros y aprendan a reconocer sus debilidades y fortalezas, siendo ellos mismos participes activos en el proceso evaluativo.

Es decir, se necesita una concepción alternativa para la eval uación de los aprendizajes que se fundamente en principios propios de la evaluación auténtica (IbarraSaiz y Rodríguez-Gómez, 2020) Concepción evaluativa ba sada en las directrices de la perspectiva práctica-critica del curriculum y rotundamente vinculada a los procesos, así como en plantear el carácter ideológico de la evaluación, bajo determinantes constructivistas y de pensamiento crítico. Evaluación que debe ser antes que nada un medio para formar al estudiante en su progreso y desarrollo del proceso de enseñanza y aprendizaje. Situándonos en un enfoque más complejo de la evalua ción: formativo-cualitativo (Golding y Adam, 2016).

Como señal aA humada (2005), la evaluación auténtica, intenta averiguar qué es capaz de hacer el estudiante, utilizando diferentes estrategias y procedimientos eval uativos. Las bases teóricas que fundamentan esta concepción tienen sus raíces como argumentan Condemarín y Medina (2000) en la concepción del aprendizaje significativo de Ausubel, en la perspectiva cognoscitivade N ovak y en laprácticareflexivadeSchön. Una concepción evaluativa desde un enfoque educativo que se cimienta, como se ha estudiado en otras investigaciones (Calatayud, 2019, 2019a), en los siguientes supuestos:

- Una evaluación en la que existe un mayor predominio de la evaluación informal frente a la evaluación formal. 
-Evaluación significativa, diversificada, diagnóstica, procesual, contextualizada, coherente, cualitativa, ideográfica y transferible.

-Evaluación integrada en el proceso de enseñanza y aprendizaje.

-Evaluación continua desde un enfoque constructivista.

-Mayor potenciación de informes cualitativos en la evaluación del aprendizaje del alumnado.

-Evaluación que plantea la necesidad de evaluar también lo positivo y no sólo lo negativo.

-Evaluación que incorpora la autoevaluación y la coevaluación.

-El trabajo del alumno como principal fuente en los procesos de evaluación del aprendizaje.

- Se evalúan tanto contenidos conceptuales, procedimentales y actitudinales.

-Evaluación con carácter implícito.

Por tanto, se trata de una evaluación que está integrada dentro del proceso de enseñanza y aprendizaje y no separada o desconectada de él. Planteamiento que se opone al que concibe la evaluación como una práctica que se efectúa al final de un periodo más o menos prolongado de enseñanza, 0 al término de la realización de alguna unidad temática mediante un acto formal explícito de comprobación, como es el poner una prueba 0 realizar un examen (Sanmartí, 2020). Se intenta que la evaluación no sólo sea un acto que conduzca a poner una nota sino que, sobre todo, ésta se entienda como un proceso de comprensión, de diálogo, de motivación ha cia el aprendizaje tanto del profesor como de los alumnos. U na evaluación, a fin de cuentas, como señalan autores como Fernández-Pérez (1986), Richard y Godbout (2000) Tolgfors y Ö hman, (2015) que eduque, que nos enseñe «qué es debido a qué», una evaluación formativa y no únicamente sumativa. En este sentido, una evalua ción para que sea realmente educativa, emancipadora e iluminativa tiene que ser concebida como:

- U na reflexión sobre los procesos de enseñanza y aprendizaje, las finalidades que los orientan y los condicionantes que confinan y restringen su desarrollo.

- Un proceso continuado de investigación queno puede entenderse únicamente como referida al aprendiza je al canzado por los estudiantes, sino que tiene que ser una valoración de todo el proceso y de todos los elementos que en él participan (docente, alumnos, asigna tura, estrategias metodológicas, etc.). Una evaluación que como señalan los autoresAllal (2010) yAllen (2000) tanto de los conocimientos, actitudes y habilidades adquiridos por los discentes y en los que la evaluación no se reduce al momento final del proceso, sino que se realiza en distintos momentos y con diversas estrategias.

Desarrollar la evaluación desde estos supuestos requiere como argumentaCalatayud (2019; 2019a) deunos requisitos, algunos de ellos son:

- U na eval uación que esté integrada en el proceso de enseñanza y aprendizaje.

- Una evaluación que incidaen el proceso, formativa, procesual, continua, etc.

- Una evaluación que se haga con la finalidad básica de obtener información del alumnado, del proceso y del contexto de aprendizaje, con el fin de mejorarlos. Q ue sea, en definitiva, útil.

- Una evaluación holística. Evaluación de conceptos, procedimientos, actitudes competencias.

- Una evaluación que reconozca los effuerzos realizados por los estudiantes.

- U na evaluación que sea respetuosa con la persona.

- U na evaluación que utilice diversidad de instrumentos para evaluar el aprendizaje del alumnado.

- U na evaluación dentro de lo posible que sea ética, basada en compromisos explícitos que aseguren la cooperación y la aceptación de las personas implicadas. En este sentido, los criterios de evaluación serán públicos, explicitados y negociados con los estudiantes.

- U na eval uación que sea coherente con los contenidos, la enseñanza y las actividades realizadas en clase («Dime cómo enseñas y te diré cómo evalúas).

- Una evaluación que presente distintos niveles de dificultad.

- Una evaluación centrada en si el estudiante es capaz de relacionar lo aprendido con otros conocimientos, si es capaz de trasladarlo a situaciones distintas a aquellas que generaron el aprendizaje original.

- U na evaluación que favorezca la reflexión, la innovación, la creatividad, así como, el pensamiento crítico y lateral.

- Una evaluación que fomente el trabajo cooperativo y las relaciones socio-afectivas.

\section{Diseño de la investigación}

El objetivo general de esta investigación era analizar las metáforas del alumnado universitario de Educa ción Física del curso académico 2019-20 acerca de la evaluación vivida. Se pretendía constatar si la evaluación que habían vivido distaba o no mucho de oscurida des relativas a control, examen, certificación, competi- 
ción, etc.

Los objetivos específicos que se querían conseguir eran los siguientes:

1-Delimitar las categorías evaluativas que caracterizaron la evaluación en los años de escolaridad.

2-Identificar qué tipos de metáforas radiografían las practicas evaluativas vividas.

3-Clasificar las metáforas propias de cada una de las Tradiciones Evaluativas: Examinadora y Educativa.

Participantes en la investigación. H an participado en este estudio 275 estudiantes del Grado de Magisterio de Educación Física de Primer curso de varias U niversida des Públicas de la Comunidad Valenciana (España) del curso 2019-20. La participación del estudiantado fue voluntariaY han participado estudiantes de laU niversitat deVal ència (225 participantes), U niversitat de Alicante (33 participantes) y la U niversitat Jaume I de Castellón (17 participantes).

Diseño de la investigación. Se utilizó una metodología cualitativa de corte hermenéutica. Se diseñó un cuestionario con una sola pregunta abierta para indagar una cuestión relativa a las metáforas evaluativas. La cuestión que se planteó al alumnado fue la siguiente: «Describe las Metáforas que mejor representan la evalua ción vivida en tus años como estudiante en Secundaria o Bachillerato». Se comentó al estudiantado que debía de nombrar las metáforas y luego describirlas e incluso si se quería se podía acompañar con un dibujo.

\section{Análisis de los datos}

Para la interpretación de las respuestas dadas a la pregunta formulada se utilizó la metodología del análisis de contenido. Posteriormente, se ha realizado un proceso de codificación y categorización de los datos recogidos. Se ha optado por no utilizar programas informáticos para su análisis. En la tabla 1 se presenta el diseño de las categorías que emerge del análisis de da tos y que hemos utilizado como estructura básica para presentar los resultados. A la hora de codificar la información se ha partido de la idea de que las metáforas planteadas por los estudiantes de Educación Física son códigos in vivo propuestos por los participantes en la investigación o incluso, al gunas de ellas, han emergido del análisis de las respuestas dadas.

\section{Tabla 1.}

Categorías elaboradas para el análisis de datos.

1-Sentimientos evaluativos: miedo, desconcierto, etc.

2-Dificultades evaluativas

3-Procedimientos evaluativos

4-Curriculum evaluador
5-Evaluación orientada al desarrollo de procesos mentales catalogados como memorización, repetición, etc.

6-Tatuaje evaluativo

7-Exigencias eval uativas

8-Evaluación orientada al control

9-Evaluación como acreditación o reconocimiento social

10-Evaluación uniformadora.

11-Stress eval uativo.

12-Evaluación como aprendizaje

\section{Resultados y discusión}

Este apartado se ha organizado en función del sistema de categorías presentado en la tabla 1. Dado que la investigación es de corte cualitativo, hemos unido en el mismo apartado la presentación de resultados y la discusión. Destacar que después de anal izar las respuestas de la muestra participante se agruparon de forma sintética las metáforas más relevantes de los estudiantes. Para el tratamiento de los datos se hizo un análisis de contenido cualitativo, organizando la información obtenida por metáforas. Hemos de matizar que las categorías han sido diseñadas por la investigadora, en cambio, las metáforas son las que han explicitado los estudiantes. En algunas de ellas, se citan palabras textuales del estudiantado.

A continuación, se describen las metáforas que se engloban en cada una de las categorías propuestas y se explican textualmente los comentarios que los estudiantes han real izado para relatar el significado de cada una de las metáforas.

\section{1-Sentimientos evaluativos: miedo, desconcier- to, etc. \\ Q ue engloba las metáforas siguientes: \\ -* La evaluación como un océano. \\ Metáfora que hace mención que al igual que en el} mar hay mucha agua, derramamos muchas lágrimas por el miedo a suspender, etc.

-* La evaluación como los desvelos del infierno.

Metáfora referida a los desvel os que se sienten antes de la fecha de los exámenes que no dejan descansar ni dormir.

-* La evaluación como cita con el médico.

Siempre cuando vas a hacer un examen vas con nervios por si sale mal, como cuando acudes a la consulta del médico dónde no sabes que te va a diagnosticar.

\section{2-D ificultades evaluativas}

Q ue engloba las metáforas siguientes:

** La evaluación como la cima del Everest. 
La evaluación es símil de lucha y esfuerzo por alcanzar la cima que es pasar el examen

-* La evaluación como un zoo de fieras, como competición.

La evaluación comportalucha, competitividad, agresiones para ver quién es el mejor. Q uien termina antes las pruebas y quien saca buenas notas.

-* La evaluación como una carrera de obstáculos.

Se compite para ver quién es el mejor y llega primero. Los exámenes son los obstáculos que te encuentras en la carrera. Algunos estudiantes lograrán superarlos sin ningún problema y otros no.

\section{3-Procedimientos evaluativos}

Q ue engloba las metáforas siguientes:

-* La evaluación a base de controles escritos.

Esta metáfora se manifiesta en aquellos docentes que utilizan un único método de eval uación a través de controles escritos. El reiterado uso de estas pruebas provoca un descenso de la motivación y acaba frustrando a aquellos estudiantes que no consiguen aprobarlos.

-* La evaluación como examen.

Se cree que cuánto más exámenes y más complica dos sean más se aprende.

-* La evaluación como un muro de conocimiento.

El hecho de evaluar con exámenes muchas veces limita el conocimiento compartido.

-* La evaluación como peces en el agua.

Los estudiantes pasan mucho tiempo sentados en la silla. Los docentes creen que estando sentados, losalumnos están aprendiendo pero esto pocas veces ocurre. Como señala un estudiante: «se pasa mucho tiempo sentado y haciendo exámenes como lo están los peces en el agua. N os olvidamos de jugar y de vivir»

-* La evaluación son exámenes que te exprimen el cerebro como una naranja.

El cerebro es la naranja y el exprimidor es la eva luación. A veces la naranja tiene más o menos jugo. En este sentido, un estudiante argumenta que «el docente no puede exprimir más si la naranja es de un 6 , y que, por mucho, que exprima no puede sacar más zumo para que sal ga un 10».

-* La evaluación como prueba objetiva.

Esta metáfora hace referencia a los exámenes de carácter objetivo. Existe la creencia que a través de este tipo de pruebas está más patente la objetividad en la evaluación.

-* La evaluación como trabajo individual.

Hace referencia a que todo se evalúa de forma individual, cada estudiante tiene su material curricular du- rante todo el curso y debe encontrar las mejores estrategias y procesos para aprobar las asignaturas contenidas en dicho material curricular.

-* La evaluación como caja de sorpresa.

Hay profesores que en los exámenes ponen ejercicios que jamás han explicado en clase. Por tanto, es imposible que los estudiantes sepan responder a esa cuestión. El examen que realizan estos docentes se puede comparar con una caja de sorpresas puesto que no se sabe lo que va a exigir el profesor en el examen.

\section{4-Curriculum evaluador}

Q ue engloba las metáforas siguientes:

-* La evaluación como una partida. De jugarse las cartas en una partida.

Se está estudiando durante mucho tiempo y como señal a un estudiante: «te lo juegas todo a una car ta en la realización de los exámenes»

-* La evaluación como un tren.

Esta metáfora incide en que el profesor conduce la locomotora con el objetivo de pasar por cada una de las estaciones (tema y su correspondiente examen) a las horas establecidas (cumplir con el temario). Cada alumno/ a se relaciona con los vagones del tren que van siguiendo a la locomotora. Además, cuando el profesor/ a corrige el examen final se da cuenta de que hay pocos estudiantes que han llegado al destino pasando por todas las estaciones. Y en contraposición, muchos se han ido perdiendo por el camino (han ido suspendiendo y no han asimilado los conceptos explicados).

-* La evaluación como un casting.

En un casting vas pasando fases, y si no superas los requisitos de una de ellas te quedas sin pasar a la siguiente. Es como cuando un estudiante suspende y tiene que hacer la recuperación o repite curso.

-* La evaluación como un reloj de arena.

Cuando se realiza un examen en la escuela, los estudiantes tienen un tiempo muy limitado, si se pasan de ese tiempo, el profesor puede llegar a no querer recoger ese examen y suspenderle. El reloj de arena simboliza el tiempo que les queda a los estudiantes para completar dicho examen.

\section{5-Evaluación orientada al desarrollo de proce- sos mentales catalogados como memorización, re- petición, etc. \\ Q ue engl oba las metáforas siguientes: \\ -* La evaluación como repetición sin sentido. \\ La evaluación que se practica no es para hacer que pienses, para desarrollar el pensamiento crítico, sino}


para escribir lo que pone el libro de texto. Se aprende repitiendo todo como si fueras un loro, sin ningún tipo de criterio propio.

-* La evaluación como un tornado.

Porque la cabeza no para de dar vueltas con tanta información que has de recordar para hacer el examen.

-* La evaluación como memoria, como un loro 0 como regurgitación cognitiva.

La evaluación consiste en memorizar y, después, soltar esa información sin aprenderla. Los profesores se centran en explicarlo todo como está en los libros de texto, lo que supone como comenta un estudiante, el que «los alumnos terminen repitiéndolo todo de la misma manera como está en el libro».

-* La evaluación como obesidad.

Esta metáfora incide según los estudiantes en que «una persona obesa come muchos alimentos y por ende acumula muchas grasas. Pero éstas no sirven para nada sólo se almacenan.» Del mismo modo ocurre con los contenidos, si no se aprenden bien, se memorizan con el único objeto de «vomitarlo» en el examen.

-* La evaluación como el «Gran Hermano».

Esta metáfora hace referencia a que la evaluación no facilita el pensamiento crítico y se considera que la valía viene determinada por una nota numérica. Y como señala una estudiante: «las personas no somos un número».

-* La evaluación como un vertedero de conocimientos.

La escuela, a través de la evaluación, funciona como una fábrica que deshecha a aquellos que no son «útiles» para la sociedad y convierte en basura a personas muy válidas sólo por el hecho de no adaptarse al perfil que se demanda.

\section{6-Tatuaje evaluativo}

Q ue engloba las metáforas siguientes:

-* La evaluación como un pozo sin fondo.

Detrás de un examen viene otro y, así sucesivamente. Como señala un estudiante, cada vez: «se hace más duro y sientes que no llegas a estudiarlo todo. Entonces, es como si todo se hiciera más oscuro y no ves ningún punto de luz que te ayude a saber que has llegado a la meta»

-* La eval uación como un combate de boxeo.

Continuamente te están evaluando y te golpean por todas partes (trabajos, exámenes, etc.) y tienes que conseguir salir victorioso.

-* La evaluación como un número de condena.

La evaluación adjudicaunanota en un momento con- creto que determina las posibilidades que el sistema educativo ofrecerá al estudiante en un futuro.

-* La evaluación como drama.

Como argumenta un estudiante: «nos ponen mucho para estudiar y ello nos da tristeza y angustia, siempre estamos sin ganas de nada y, por ello, el resultado muchas veces no es el esperado»

-* La evaluación como un burro.

A causa de los malos resultados en los exámenes, tus compañeros te llaman burro ya para siempre.

-* La evaluación según el apellido.

Esta metáfora incide en que muchos docentes, califican los exámenes teniendo en cuenta quienes son los estudiantes. Así lo comenta una alumna: «los exámenes los califican según el apellido. Te suben 0 te bajan la nota, según si caes bien o mal al docente».

-* La evaluación como la mordaza en la silla.

Esta metáfora toma como afirmación la siguiente idea planteada por ejemplo, por un estudiante: «la eva luación te ata de pies y manos y no puedes hacer otras cosas»

\section{7-Exigencias evaluativas}

Q ue engloba las metáforas siguientes:

-* La evaluación como un infierno.

Todas las semanas tienes exámenes y no se acaba nunca.

-* La evaluación como una barrera que me separa de mis pasatiempos

La eval uación ocupa demasiado tiempo en nuestras vidas, no dejando tiempo para jugar. En este sentido, una estudiante señala que la evaluación condiciona el que no puedas «hacer lo que realmente te gusta». La evaluación nos separa de nuestra vida privada y se convierte en nuestra única prioridad»

-* La evaluación como avalancha.

Esta metáfora se refiere a la cantidad de tareas que los estudiantes han de realizar para pasar los exámenes (deberes, actividades, exposiciones, etc.)

\section{8-Evaluación orientada al control}

Q ue engloba las metáforas siguientes:

-* La evaluación como una prisión.

La evaluación es como una cárcel en la que nos sentimos encerrados, tanto en las metodologías impuestas como en la realidad que nos muestran y que quieren que veamos y creamos. Y, por supuesto, como afirma un estudiante: «después se ha de vomitar»

-* La evaluación como comparación.

M etáfora que sucede en aquellos casos donde el pro- 
fesor hace comparaciones con sus estudiantes de las notas que sacan en los exámenes delante de toda la clase, lo que conlleva una repercusión negativa y de inferioridad en aquellos discentes que tienen notas más bajas.

\section{9-Evaluación como acreditación o reconocimien- to social}

Q ue engloba las metáforas siguientes:

-* La evaluación como una nota que define a la persona.

Vales según la nota que saques en los exámenes.

-* La evaluación únicamente del resultado.

El docente sólo refleja en la evaluación del estudiante el resultado obtenido en los exámenes o en los controles, sin importarle el esfuerzo, el interés o la actitud del alumno por la asignatura.

* La evaluación como la marca de la valía.

Se entiende la evaluación como una selección de los que valen y de los que no valen.

-* La evaluación como tocar el cielo con las manos.

Cuando al final de curso consigues las calificaciones deseadas teinundas de al egría y tal como señala un al umno: «es como si tocaras el cielo con las manos»

-* La evaluación como un análisis de sangre.

Tanto el análisis de sangre como la evaluación te dice qué asignaturas llevas aprobadas y cuáles te han salido mal.

** La evaluación como un colador.

La evaluación actúa como un colador. Con este instrumento se separa a los estudiantes que no están en el nivel adecuado de los que sí. M oviendo el colador, caen por los agujeros los alumnos que no están en el nivel correcto.

-* La evaluación como una selva.

Donde el animal más fuerte (lo comparamos con el estudiante que tiene pocas dificultades en las asignaturas) domina a aquel animal más débil (comparado con el estudiante que necesita un refuerzo especifico o una adaptación del currículo a sus necesidades y habilidades) obteniendo así una recompensa mejor.

\section{0-Evaluación uniformadora}

Q ue engloba las metáforas siguientes:

-* La evaluación como un rebaño de ovejas.

La evaluación se asemeja a un rebaño de ovejas ya que no se tiene en cuenta al individuo. Además, no se centran en las capacidades, ni ritmos de cada estudiante. $N o$ se atiende a la diversidad.

-* La evaluación como una escalera.

$\mathrm{H}$ ay que ir subiendo los escalones. Si pasas la prueba subes y si no la pasas te quedas en el mismo escalón.

-* La evaluación como un ascensor.

Con respecto a las calificaciones tienes que conseguir llegar al último piso y hacerte con la nota máxima y saber mantenerte.

-* La evaluación como un martillo.

Se compara a cada alumno con un clavo y la evaluación con un martillo. Cada discente posee un potencial en su interior y el hecho de que saque un 0 en un exa men no quiere decir que ese estudiante no valga para nada.

-* La evaluación como un bosque que no deja ver el árbol.

Muchas veces los profesores enseñan y, por lo tanto, evalúan para una media que realmente no existe. De este modo le damos la vuelta a la metáfora, siendo el bosque la media y el árbol el estudiante.

-* La evaluación del pez mono.

Evaluación similar para todos a pesar de que todos somos diferentes.

-* La evaluación como una media.

Esta metáfora quiere decir que la mayoría de los profesores sólo se fijan en las notas de las pruebas y deberes, es decir, calculan la media y ya está, sin observar la evolución de cada alumno, la mejora de la actitud, etc.

-* La evaluación como robots.

Los profesores muchas veces tienen como objetivo formar a personas iguales. En los exámenes hay docentes que exigen a los estudiantes realizar los ejercicios exactamente como ellos han explicado en la pizarra las semanas antes del examen, sin embargo, puede ser que los estudiantes puedan hacerlo de forma diferente pero que lleguen al mismo resultado. Estos profesores suelen penalizar dicha acción.

\section{1-Stress evaluativo}

Q ue engloba las metáforas siguientes:

-* La evaluación como estrés.

Esta metáfora incide en la idea de la cantidad de materia que va para un examen. Muchas veces, como señala una estudiante el «ver que tienes mucha materia que estudiar y no tienes tiempo, hace que te provoque estrés, depresión, angustia, etc.

-* La evaluación como temblor cardio respiratorio.

Cuando se acercan las fechas de los exámenes aparece el nerviosismo y, como señala una estudiante: «ves que se te acelera el ritmo cardiaco, las pulsaciones, los nervios, etc.»

-* La evaluación como un nudo marinero en el estó- 
mago.

El enorme dolor que se siente en el estómago cuando se acerca la evaluación. Según un alumno es: «como si se tuviera un nudo en el estómago.».

-* La evaluación como película de miedo.

Esta metáfora incide en que todo el proceso de eva luación es como si fuera una película de miedo. Siempre estás en tensión, en pánico, etc.

\section{2-Evaluación como aprendizaje}

Q ue engloba las metáforas siguientes:

-* La evaluación como aprendizaje constante.

La eval uación te ayuda a aprender de tus errores. Se utiliza la evaluación como una fuente de aprendizaje.

-* La evaluación como mejora interna personal.

La evaluación te ayuda a mejorar no sólo los rendimientos sino también aspectos más personales (tener

\begin{tabular}{|c|c|}
\hline Categorías & Metáforas evaluativas \\
\hline 1-Sentimientos eval uativos. & $\begin{array}{l}\text { - la evaluación como un océano. } \\
\text {-la evaluación como los desvel os del infierno. } \\
\text {-la evaluación como cita con el médico. }\end{array}$ \\
\hline 2-Dificultades evaluativas & $\begin{array}{l}\text { - la evaluación como la cima del Everest. } \\
\text { - la evaluación como un zoo de fieras, como competición. } \\
\text {-la evaluación como una carrera de obstácul os. }\end{array}$ \\
\hline 3-Procedimientos eval uativos & $\begin{array}{l}\text { - la evaluación a base de controles escritos. } \\
\text { - la evaluación como examen. } \\
\text { - la evaluación como un muro de conocimientos. } \\
\text { - la evaluación como peces en el agua. } \\
\text { - la evaluación son exámenes que te exprimen el cerebro como } \\
\text { una naranja. } \\
\text { - la evaluación como prueba objetiva. } \\
\text {-la evaluación como trabajo individual. } \\
\text {-la evaluación como caja de sorpresa. }\end{array}$ \\
\hline 4-Curriculum evaluador & $\begin{array}{l}\text { - la evaluación como una partida. } \\
\text { - la evaluación como un tren. } \\
\text { - la evaluación como un casting. } \\
\text { - la evaluación como un reloj de arena. }\end{array}$ \\
\hline $\begin{array}{l}\text { 5-Evaluación orientada al } \\
\text { desarrollo de procesos } \\
\text { mentales catal ogados como } \\
\text { memorización, repetición, } \\
\text { etc. }\end{array}$ & $\begin{array}{l}\text { - la evaluación como repetición sin sentido. } \\
\text { - la evaluación como un tornado. } \\
\text { - la evaluación como memoria, como un loro o como } \\
\text { regurgitación cognitiva. } \\
\text { - la evaluación como obesidad. } \\
\text { - la evaluación como el gran hermano. } \\
\text { - la evaluación como un vertedero de conocimiento. }\end{array}$ \\
\hline 6-Tatuaje eval uativo & $\begin{array}{l}\text { - la evaluación como un pozo sin fondo. } \\
\text { - la evaluación como un combate de boxeo. } \\
\text { - la evaluación como un número de condena. } \\
\text { - la evaluación como drama. } \\
\text { - la evaluación como un burro. } \\
\text { - la evaluación según el apellido. } \\
\text { - la evaluación como la mordaza en la silla. }\end{array}$ \\
\hline 7-Exigencias evaluativas & $\begin{array}{l}\text { - la evaluación como un infierno. } \\
\text { - la evaluación como una barrera que me separa de mis } \\
\text { pasatiempos. } \\
\text { - la evaluación como aval ancha. }\end{array}$ \\
\hline $\begin{array}{l}\text { 8-Evaluación orientada al } \\
\text { control }\end{array}$ & $\begin{array}{l}\text { - la evaluación como una prisión. } \\
\text {-la evaluación como comparación. }\end{array}$ \\
\hline $\begin{array}{l}\text { 9-Evaluación como } \\
\text { acreditación o } \\
\text { reconocimiento social }\end{array}$ & $\begin{array}{l}\text { - la evaluación como una nota que define a la persona. } \\
\text { - la evaluación únicamente del resultado. } \\
\text { - la evaluación como la marca de la valía. } \\
\text { - la evaluación como tocar el cielo con las manos. } \\
\text { - la evaluación como un análisis de sangre. } \\
\text { - la evaluación como un col ador. } \\
\text { - la evaluación como una selva. }\end{array}$ \\
\hline 10-Eval uación uniformadora & $\begin{array}{l}\text { - la evaluación como un rebaño de ovejas. } \\
\text { - la evaluación como una escalera. } \\
\text { - la evaluación como un ascensor. } \\
\text { - la evaluación como un martillo. } \\
\text { - la evaluación como un bosque que no deja ver el árbol. } \\
\text { - la evaluación del pez mono. } \\
\text { - la evaluación como una media. } \\
\text {-la evaluación como robots. }\end{array}$ \\
\hline 11-Stress evaluativo & $\begin{array}{l}\text { - la evaluación cono stress } \\
\text { - la evaluación como temblor cardio-respiratorio. } \\
\text { - la evaluación como un nudo marinero en el estómago. } \\
\text { - la evaluación como película de miedo. }\end{array}$ \\
\hline $\begin{array}{l}\text { 12-Eval uación como } \\
\text { aprendizaje }\end{array}$ & $\begin{array}{l}\text { - la evaluación como aprendizaje constante. } \\
\text {-la evaluación como mejora interna personal. }\end{array}$ \\
\hline
\end{tabular}

constancia, esfuerzo, etc.).

Como resumen en laTabla 2 se presenta el sistema de categorías y de metáforas que emergen del análisis de datos y que hemos utilizado como estructura básica para presentar los resultados.

Desde la mirada de los estudiantes hemos constata do la existencia de 57 metáforas evaluativas que radiografían la evaluación vivida. Como se puede observar las 57 metáforas se han agrupado en 12 categorías y sólo hay una que puede englobarse en las coordinadas de la Tradición Educativa. Las 55 metáforas restantes forman parte de la cultura evaluativa inmersa en la Tradición Examinadora de la evaluación. Constatar esta realidad es necesario para darse cuenta de que ésta ha de cambiar si queremos realizar prácticas evaluativas de excelencia que estimulen la generación de aprender a aprender y del uso de la evaluación como fuente de aprendizaje. Los docentes universitarios tenemos el reto de formar a futuros profesores que sean promotores del cambio, la innovación evaluativa y de la concepción de la evaluación como una oportunidad para la mejora y el aprendizaje.

Como se puede observar, los resultados obtenidos en esta investigación, reflejan que hay unas categorías más próximas a una tradición u otra, tal y como hemos explicitado en el punto 2 y 3 . Desde el discurso plantea do en esos apartados, las categorías evaluativas propias de laTradición Examinadora son las siguientes:

1-Sentimientos evaluativos: miedo, desconcierto, etc.

2-D ificultades evaluativas

3-Procedimientos evaluativos

4-Curriculum evaluador

5-Evaluación orientada al desarrollo de procesos mentales catalogados como memorización, repetición, etc.

6-Tatuaje eval uativo

7-Exigencias evaluativas

8-Evaluación orientada al control

9-Evaluación como acreditación o reconocimiento social

10-Evaluación uniformadora.

11-Stress evaluativo.

Y en la Tradición Educativa de la eval uación, la categoría es la siguiente:

12-Evaluación como aprendizaje

\section{Consideraciones finales}

A lo largo de este articulo hemos analizado las metáforas del alumnado universitario de Educación Física acerca de la evaluación vivida. Se pretendía constatar si la evaluación que habían vivido distaba o no mucho de 
oscuridades relativas a control, examen, certificación, competición, más propias de laTradición Examinadora ver susTradición Educativa. Los objetivos específicos que se querían conseguir eran los siguientes:

1-Delimitar las categorías eval uativas que caracterizaron la evaluación en los años de escolaridad.

2-Identificar qué tipos de metáforas radiografían las practicas evaluativas vividas.

3-Clasificar las metáforas propias de cada una de las Tradiciones Eval uativas: Examinadora y Educativa.

Después de los resultados extraídos en esta investigación, una de las cuestiones que ha quedado suficientemente clara es que asistimos a una cultura evaluativa vivida dentro de las coordenadas de lo que hemos denominado Tradición Examinadora de la evaluación. U na cultura desfasada y que no se adapta al escenario en el que estamos viviendo. Resulta muy preocupante el que la evaluación sólo despierte mayoritariamente metáforas propias de laTradición Examinadora. De las 57 sólo 2 metáforas inciden en mejora, aprendizaje, superar dificultades, reflexión, crecimiento, ayuda, etc.

Indudablemente, un anál isis de las metáforas expuestas conlleva la necesidad de reinventar la evaluación que practicamos a los estudiantes. Ésta ha de cambiar y empezar a reinventar una concepción de la evaluación asentada en supuestos formativos para que luego cuando sean profesores de Educación Física puedan trasladar a sus alumnos y alumnas. Evaluación que facilitará la mejora de los aprendizajes de los estudiantes, potenciando al máximo sus capacidades y sus posibilidades y en la que se utilizará el error como fuente de aprendizaje y en la que la retroalimentación presenta una gran relevanciaparalamejora del proceso educativo. (Hernán et al. , 2020: López-Pastor et al. , 2016; Rodríguez-Gómez y lbarra-Sáiz, 2015).

Ante esta situación imperante es necesario plantear estrategias de actuación que ayuden a superar esta tra dición Examinadora de la evaluación asentada en parámetros de control y examen. Por ello, si tuviéra mos que jerarquizar los dos aspectos prioritarios sobre los que se debería de incidir para posibilitar un cambio metafórico de la evaluación, serían básicamente los siguientes:

A-Potenciar una formación en evaluación a los estudiantes de Educación Física que incida en los siguientes principios que autores como Ahumada (2005), Anijovich y Cappelletti (2017) señalan:

1-Continuidad y permanencia de la evaluación: Este principio incide en que hoy por hoy la evaluación debe consistir en un proceso más que en un suceso y, por tanto, interesa obtener evidencias centradas más en los procesos de aprender que en los resultados o productos de los aprendizajes.

2-Del carácter retroalimentador del proceso evaluativo. Incide este principio en aceptar la presencia del error como una forma natural de aprender.

3-Del carácter activo y participe del alumnado en el proceso evaluador. El estudiante ha de participar en su evaluación a través de procesos autoevaluativos, coevaluativos, etc.

B-El uso de nuevos procedimientos evaluativos para potenciar una evaluación auténtica en la Educación Física. A continuación, se presentan una serie de procedimientos, siendo una superación de lospresentadosen Calatayud (2019; 2019a):

* Actividades prácticas que pretendan fomentar en los estudiantes la capacidad de juicio autónomo, de disentir y discrepar, de buscar soluciones personales a los problemas. No se puede caer en la trampa, de la respuesta convergente y correcta. Desde esta visión, se han de proponer a los estudiantes un sistema de trabajo que les permita desplegar su capacidad de comprensión y reflexión sobre las ideas expuestas en clase y las lecturas realizadas.

* Pruebas de aprendizaje con el objeto de conocer si los estudiantes comprenden y transfieren los contenidos fundamentales de cada uno de los temas. Instrumentos que recojan, por un lado, la sistematización de los conocimientosy, por otro, su organización interna. Para ello, es necesario el que trabajen a partir de mapas conceptuales, rúbricas, etc.

* Trabajos de investigación Se pretende que cumplan la misión de indagación, de investigación, etc. Se valora el rigor en las argumentaciones, la coherencia, la capacidad de reflexión crítica, la utilización de bibliografía adecuada, el uso del lenguaje correcto, la presentación, etc.

* El portafolio, instrumento para la evaluación formativa del estudiante. El portafolio es un método de evaluación del alumnado, alternativo a los métodos tradicionales. Viene a ser como una colección de trabajos, activida des, etc. que el estudiante ha realizado durante un curso. Q uizás, lo más importante de esta selección de actividades radique en las siguientes premisas: deben de tratar de evidenciar los esfuerzos realizados por el estudiante, la valoración del trabajo conseguido (¿qué sa bia?, ¿qué se yo ahora?, ¿cómo lo he aprendido?, en relación a contenidos tanto del ámbito conceptual, procedimental y actitudinal, así como, las competencias aprendidas), cuáles han sido las mejores ideas, los 
logros conseguidos en los distintos ámbitos de conocimiento y, sobre todo, el portafolio debe de estar compuesto por aquellas actividades que han permitido al estudiante tanto la posibilidad de val orarse más a sí mismos, como de sentirse más seguros de sí mismos (Boud, 1995).

Indudablemente, esta forma de practicar la evalua ción conlleva necesariamente una perspectiva también distinta de pensar y desarrollar el proceso educativo. Implica una metodología basada en el aprendizaje constructivo, en el fomento de la creatividad, la reflexión, la colaboración, la actividad, la participación, etc. que posibilite al alumno progresar en su madurez y conseguir un equilibrio personal y una integración social.

* Las rúbricas.

Este instrumento nos va a facilitar el aprendizaje, dado que determina qué se espera y a su vez, constituye una guía de evaluación tanto para el alumnado como para el profesorado.

* Dianas de evaluación

Q ue nos ayudan a averiguar cómo está aprendiendo el estudiante, especificando de forma clara qué se quiere evaluar y los indicadores que se van a utilizar.

* Kit-Kat evaluativo.

Se trata de plantear preguntas relámpago de retroa limentación para asegurarse que los estudiantes están comprendiendo el mensaje durante el desarrollo del proceso de enseñanza y aprendizaje.

* Diario de aprendizaje

Instrumento que desarrolla la metacognición en el al umnado. Cada estudiante reflexiona sobre lo aprendido, qué dificultades ha tenido, qué puede mejorar, etc.

* Planing evaluativo.

Esta técnica consiste en resaltar los aspectos más importantes que se van a trabajar durante una sesión, para que los estudiantes sepan en cada momento lo que van a dar 0 trabajar.Y al final de la sesión se realiza una pequeña recapitulación evaluativa para conocer si lo pretendido al inicio se ha desarrollado y se ha aprendido de forma significativa.

Como conclusión señal ar que desde la mirada de los estudiantes universitarios de Educación Física podemos constatar que las metáforas evaluativas distan mucho del paradigma educativo centrado en el aprendizaje y en la persona que aprende. Constatar esta realidad ha sido necesario para darse cuenta de que ésta ha de cambiar si queremos realizar prácticas eval uativas de excelencia que estimulen la generación de aprender a aprender y del uso de la evaluación como fuente de aprendizaje. N os ha proporcionado una serie de puntos de re- flexión sobre el conocimiento evaluativo imperante en los estudiantes universitarios de Educación Física y en la necesidad de plantear alternativas para ir más allá de una evaluación basada exclusivamente en la calificación que sólo pretende aprobar y certificar (López-Pastor, 1999, 2006, 2008; López-Pastor et al. , 2013; RodríguezGómez. y lbarra-Sáiz, 2015; M acPhail \& M urphy, 2017).

Los docentes universitarios tenemos el reto de formar a futuros profesores que sean promotores del cambio no sólo de concepcionessino de prácticas evaluativas. Ello supone una exigencia y una necesidad hoy en la profesión docente. Exigencia y necesidad que debería regirse por intentar dar respuesta a estas cuestiones:

* ¿Por qué es excluida la evaluación como actividad de aprendizaje, de compresión, de mejora, de ayuda?

* ¿Cómo se han ido configurando estas metáforas tan negativas en torno a la evaluación?

* ¿Por qué no se ha incidido en el val or educativo de la evaluación?

* ¿Q ué papel ha tenido la escolarización obligatoria de estos estudiantes en el desarrollo, configuración o pervivencia de estas metáforas examinadoras de la eva luación?

Éstas y otras preguntas nos vienen a la mente después deanalizar los resultados de estainvestigación, pero, quizás, lo que es verdaderamente importante que dejemos muy claro es que los datos obtenidos nos indican que aún falta un largo camino por recorrer para llegar a una consideración de la evaluación como mejora, superar dificultades, reflexión, crecimiento, ayuda y como oportunidad para aprender.

Esta forma distinta de vivir la evaluación ha de pasar necesariamente por una sólida formación en evaluación y por el cambio de determinadas prácticas evaluativas que han de incluir otros instrumentos alternativos de evaluación del proceso de enseñanza y aprendizaje. Estos dos aspectos son necesarios para posibilitar el que la evaluación no se asocie a metáforas como examen, memoria, agobio, etc. Todo un reto para los profesiona les de la educación comprometidos con la metáfora de la evaluación entendida como una oportunidad para el aprendizaje, la mejora y el crecimiento personal. Metáfora que tenemos que afianzar en nuestras aulas universitarias para que el alumnado de Educación Física, se empape de su cultura evaluativa y luego pueda ponerla en práctica con sus estudiantes. Todo un reto para los profesionales de la Educación Física que entienden que la evaluación es el principal motor del aprendizaje y una oportunidad para mejorar la práctica docente. Ante esta premisa nos surge esta pregunta: ¿a qué están es- 
perando los profesionales de la Educación Física para hacerla realidad?

\section{Referencias}

A humada, P. (2005). La evaluación auténtica: Un sistema para la obtención de evidencias y vivencias de los aprendizajes. Revista Perspectiva Educacional. 1 semestre, 11-24.

Allal, L. (2010). Assessment and the regulation of learning. En P. Peterson et al, International Enciclopedia of Education. Oxford: Elsevier.

Allen, D. (2000). La evaluación del aprendizaje de los estudiantes. Barcelona: Paidós.

Anijovic, R \& Cappelletti, G. (2017) La evaluación como oportunidad. BuenosAires: Paidós.

Boud, D. (1995). Enhancing learning through self assessment. London: Kogan.

Calatayud Salom, M.A (2019a). U na oportunidad para avanzar haciala evaluación auténticaen Educación Física. RETOS. NuevasTendencias en Educación Física, Deportey Recreación, 36, enero-junio, 259-265.

Calatayud Salom, M. A. (2019). O rquestar la eval uación inclusiva en los centros educativos. ¿Por dónde empezar? Revista Internacional de Educación para la Justicia Social Vol. 8, 2, 2, 165-17.

Casbon, C. \& Spackman, L. S. (2005). Assessment for learning in Physical Education. Leeds, BAALPE.

Condemarín, M \&M edina, S. (2000). Evaluación auténtica de los aprendizajes Chile.:Andrés Bello.

Fernández Pérez, M. (1986). Evaluación y cambio educativo. El fracaso escolar. Madrid: Morata.

Golding, C y\& Adam, L. (2016). Evaluate to improve: usuful approaches to student evaluation. Assessment and Evaluation in Higher Education, 41,1-14

González-Sanmamed, M et al. (2018). Ecologías de aprendizajeen la era digital: desafíos parala educación superior. Revista Publicaciones, 8, 11-38.

Hay, P. J. (2006). Assessment for learning in Physical Education. En: D. Kirk, D. M acD onald \& M. O 'Sullivan. (Eds), The handbook of Physical Education (pp. 312-325). London, England: Sage.

Hernan, E \& López-Pastor,V M . \& Brunicardi, D. (2019). ¿Por qué hago evaluación formativa y compartida y/ 0 evaluación para el aprendizaje en E.F? La influencia de la formación inicial y permanente del profesorado. RETOS. NuevasTendencias en Educación Física, Deporte y Recreación, núm.36, enero-junio, 37-43.

Hernan, E \& López-Pastor, V M \& \& Brunicardi, D. (2020). Evalua ciónAuténtica y Evaluación 0 rientadaal A prendizaje en Educación Superior. UnaR evisión en Bases de Datos Internaciona les. Revista Iberoamericana de Evaluación Educativa. 13 (2). 6783. 10.15366/ riee2020.13.2.004.

Ibarra, M.S\& Rodríguez, G. (2020). Aprendiendo a evaluar para aprender en la Educación Superior. Revista Iberoamericana de Evaluación Educativa, 13 (1),5-8.

Leirhaug, P. E. \& MacPhail, A. (2015). It's the other assessment that is the key: three N orwegian physical education teachers' engagement (or not) with assessment for learning. Sport, Education and Society, 20(5), 624-640. doi:10.1080/ 13573322.2014.975113.

López Pastor, V. M. (2008). Implementing a Formative and Shared
Assessment System in Higher Education Teaching. European Journal of Teacher Education, 31(3), 293-311.

López Pastor,V; M olina Soria, M; Pascual Arias, C; M anriqueA rribas, J. (2020). Laimportancia de utilizar evaluación formativa y compartidaen laformación inicial del profesorado de educación física: los proyectos de aprendizaje tutorado como ejemplos de buena práctica. RETOS NuevasTendencias en Educa ción Física, Deportey Recreación, núm. 37, enero-junio, 620627.

López Pastor, V; Pérez Brunicardi, D; M anriqueArribas, J ;M onjas Aguado, R. (2016). LosretosdelaEducación Física en el Siglo XXI RETOS. NuevasTendencias en Educación Física, Deporte y Recreación, núm. 29, enero-junio, 182-18

López-Pastor,V. \& Sicilia-Camacho,A. (2017). Formative and shared assessment in Higher education. Lessons learned and challenges for the future. Assessment and Evaluation in Higher Education, 42(1), 77-97. https:/ / doi.org/ 10.1080/ 02602938.2015 .1083535

López-Pastor, V. M. (1999). Prácticas de Evaluación en Educación Física: estudio de casos en primaria, secundaria y formación del profesorado. Valladolid, Universidad deValladolid.

López-Pastor, V. M. (coord.) (2006). La Evaluación en Educación Física: Revisión de los modelos tradicionales y planteamiento de una alternativa: La evaluación formativa y compartida. Buenos Aires: Miño y Dávila.

López-Pastor,V. M., Kirk, D., Lorente-Catalán, E., M acPhail, A. \& Macdonald, D. (2013). Alternative Assessment in Physical Education: A Review of International Literature. Sport, Education \& Society, 18(1), 57-76. doi:10.1080/ 13573322.2012.713860.

Lorente, E., \& Kirk, D. (2013). Alternative democratic assessment in PETE: an action-research study exploring risks, challenges and solutions. Sport, Education and Society,18(1), 77-96.

MacPhail, A. \& Halbert, J. (2010).Wehad to do intelligent thinking during recent PE': Students' and teachers' experiences of assessment for learning in post-primary physical education. Assessment in Education, 17(1), 23-39.

MacPhail, A., \& Murphy, F. (2017). Too much freedom and autonomy in the enactment of assessment? Assessment in physical education in Ireland. Irish Educational Studies, 1-16.

Ni Chróinín, D. \& Cosgrave, C. (2013). Implementing formative assessment in primary physical education: teacher perspectives and experiences. Physical Education and Sport Pedagogy, 18(2), 219-233. doi:10.1080/ 17408989.2012.666787.

Richard, J. F. \& Godbout, P. (2000). FormativeAssessment as an integral part of the teaching-learning process. Physical and Health Education Journal, $66(3), 4-9$.

Rodríguez-Gómez, G. \& Ibarra-Sáiz, M. S. (2015). A ssessment as Learning and Empowerment: Towards Sustainable Learning in Higher Education. En M. Peris-O rtizyJ. M. M erigó Lindahl (Eds.), Sustainable Learning in Higher Education. Developing Competencies for the Global Marketplace(1-20). Springer International Publishing https:/ / doi.org/ 10.1007/ 978-3-31910804-9 1

Sanmartí, N (2020). Evaluar y aprender: U n único proceso. Barcelona: 0 ctaedro.

Tolgfors, B. \& O hman, M . (2015). The Implications of Assessment for Learning in Physical Education and Health. European Physical Education Review 22(2), 150-166. 